\title{
Quantum rolling tachyon
}

\author{
Jan Ambjørn ${ }^{a, c}$ and Romuald A. Janik ${ }^{b}$, \\ ${ }^{a}$ The Niels Bohr Institute, Copenhagen University \\ Blegdamsvej 17, DK-2100 Copenhagen Ø, Denmark. \\ email: ambjorn@nbi.dk \\ ${ }^{b}$ Institute of Physics, Jagellonian University, \\ Reymonta 4, PL 30-059 Krakow, Poland. \\ email: janik@th.if.uj.edu.pl \\ ${ }^{c}$ Institute for Theoretical Physics, Utrecht University, \\ Leuvenlaan 4, NL-3584 CE Utrecht, The Netherlands.
}

\begin{abstract}
We consider the quantum treatment of the rolling tachyon background describing the decay of D-branes in the limit of weak string coupling. We focus on the propagation of an open string in the fluctuating background and show how the boundary string action is modified by quantum effects. A bilocal term in the boundary action is generated which, however, does not spoil the vanishing of the $\beta$ function at one loop. The propagation of an open string for large times is found to be very strongly suppressed.
\end{abstract}




\section{Introduction}

D-branes are maybe the most fascinating and still somewhat mysterious objects in string theory. In particular the precise quantum nature of the branes is not entirely clear: in perturbative string theory they appear as boundary conditions for the open string, while in the low energy effective field theories or string field theory (SFT) they appear as solitonic solutions to the equations of motion. The analogy with solitons in ordinary quantum field theory is in this respect slightly misleading, since solitons there never appear as additional degrees of freedom. From the SFT perspective one could argue similarly, yet there are some glitches in the picture - attempts to find a solution of SFT describing two D25 branes have failed so far [1]. So it is not clear what is the complete set of degrees of freedom. Indeed sometimes it can be convenient to use the solitons as alternative degrees of freedom instead of the fundamental fields. However, from the point of view of perturbative string theory, D-branes seem more like heavy (charged) particles which in the end have to be included as full quantum fields in a consistent quantum theory. The question of how in detail to quantize the D-branes is still open but has to be eventually addressed in order to have a complete theory.

One meets (mild) versions of the quantum nature of the D-branes for instance in the study of D-brane recoil, but also in the toy models encountered in noncritical string theory for $c \leq 1$. In particular the case $c=1$ has been much studied since it corresponds to $2 \mathrm{~d}$ critical string theory in a linear dilaton background, and the D0 branes, as noted in [2,3], can be viewed as the eigenvalues in the $c=1$ matrix model. With this interpretation the decay of an (unstable) D0-brane of the $2 \mathrm{~d}$ bosonic string theory is described by a tachyon moving in a potential (we choose $\alpha^{\prime}=1$ )

$$
V=-\frac{T^{2}}{2}
$$

and the quantum description of a single D0-brane is dictated by the Hamiltonian

$$
H=-\frac{1}{2} \frac{d^{2}}{d T^{2}}+V(T), \quad V(T)=-\frac{1}{2} T^{2}
$$

The $c=1$ matrix model can be used to analyze the decay of the D0-brane into closed string tachyons. In this calculation (as in Sen's original calculation in critical string theory) one only uses the classical aspects of the open string theory, here specifically that a classical solution in the potential (1) is given by

$$
T_{c l}(t)=T_{0} \cosh t
$$

This is an exact counterpart of the rolling tachyon background [4] in critical bosonic strings in 26 dimensions. 
In fact, in such a calculation one finds that the number of emitted (closed string) particles diverges logarithmically and the expectation value of the emitted energy diverges linearly. This is true for the decay of D0-branes both in the $c=1$ theory and in critical string theory. In the $c=1$ theory the situation could be analyzed further since the quantum theory of the D0-branes is known. Clearly the classical picture of D0-brane decay is only approximate, limited by the uncertainty principle, and it was suggested in [2] that by considering wave packets rather than the localized eigenvalues corresponding to (3) one would avoid the divergence caused by the classical open string background. This was verified in detail in [5] and in this article we discuss the extension of the results in [5] to critical bosonic string theory.

\section{The quantum boundary action}

Let us first review the $2 \mathrm{~d}$ calculation. The amplitude for emission of closed string tachyons is given by an expression

$$
A(E) \sim \lim _{l \rightarrow 0} \text { (leg factor) } \int d t e^{i E t}\left\langle\operatorname{tr} e^{-i l \Phi(t)}\right\rangle_{M}
$$

where the so called "leg factor" is unimportant to us and where

$$
\left\langle\operatorname{tr} e^{-i l \Phi(t)}\right\rangle_{M} \equiv \int d T \rho(T, t) e^{-i l T},
$$

$\Phi$ is a $N \times N$ matrix (where $N \rightarrow \infty$ ) and $\rho(T, t)$ is the eigenvalue density of $\Phi$. The contribution from the classical rolling tachyon is obtained by the substitution $\rho(T, t) \rightarrow \delta\left(T-T_{c l}(t)\right)$. Thus one ends up calculating the integral

$$
\int d t e^{i E t} e^{-i l T_{c l}(t)} .
$$

As shown in [5] this integral gave precisely the closed tachyon emission amplitude (4) obtained using continuum BCFT methods in the case of a decaying classical D0-brane. But in fact the true physical object is the quantum D0-brane, which is described by a wavefunction $\psi(T, t)$ - satisfying the appropriate Schroedinger equation. The closed string emission can then be calculated using the replacement

$$
e^{-i l T_{c l}(t)} \rightarrow\left\langle e^{-i l T}\right\rangle_{\psi(t)} \equiv \int d T|\psi(T, t)|^{2} e^{-i l T}
$$

Let us now turn to the calculation of the decay of D-branes in critical (bosonic) string theory. The dominant source of D-brane instability is the tachyon field 
mode $T(t, \vec{x})$. It lives on the boundary of the world sheet and gives rise to a boundary action

$$
S_{b}=\int_{B} d \tau T(t, \vec{x})
$$

where the integration is along the string boundary. Let us expand the tachyonic field in momentum modes, where for simplicity we compactify the spatial directions to a box with volume $V=L^{p}$ for a Dp-brane:

$$
T(t, \vec{x})=\frac{1}{(2 \pi L)^{\frac{p}{2}}} \sum_{\vec{n}} T_{\vec{n}}^{c}(t) \cdot \cos \left(2 \pi n \frac{x}{L}\right)+T_{\vec{n}}^{s}(t) \cdot \sin \left(2 \pi n \frac{x}{L}\right)
$$

We now need the spacetime action for the tachyon field. We will consider just the free quadratic part of Witten's OSFT, thus we assume that $g_{s}$ is small. We will then treat this spacetime action quantum-mechanically. We still expect nontrivial quantum effects even using just the free SFT. Indeed in [5], in the $2 \mathrm{~d}$ string case, we observed that quantum effects were very important even before including the effects of string interactions (the Fermi sea).

Inserting this decomposition into the quadratic string field theory action one obtains the following:

$$
S_{T}=\sum_{\vec{n}} \int d t\left\{\frac{1}{2} \dot{T}_{\vec{n}}^{c, s}(t)^{2}-\frac{1}{2}\left(k_{\vec{n}}^{2}-1\right) T_{\vec{n}}^{c, s}(t)^{2}\right\}
$$

Thus we get a set of inverted harmonic oscillators (with $k^{2}<1$ ) and a set of normal ones (modes with $k^{2}>1$ ). This is just the linearized approximation to a genuine string field theoretical description but will be sufficient for our purpose.

The dominant unstable mode is the spatial constant mode, so we consider first that mode. We will return to the nonconstant modes later in the paper. The (now) standard calculation of closed string emission assumes that we have a classical tachyonic background $T_{c l}(t), T_{c l}(t)$ being a solution to the classical equation originating from the truncated action (10). Such solutions are of the form

$$
T_{c l}(t)=T_{0} \cosh t \quad \text { or } \quad T_{c l}(t)=T_{0} e^{t} .
$$

In all calculations so far, whether one uses string field theory and the formalism of boundary states [4] or the conceptually simpler open string perturbative approach of [6], one always treats the tachyon profile as given by (11), i.e. as being strictly classical.

As mentioned above, one lesson from the $2 \mathrm{~d}$ critical string theory was that one has eventually to promote the classical tachyonic background to a quantum state. One (first quantized) way to do that is to replace the classical solution with a quantum wave function:

$$
T_{c l}(t) \rightarrow \psi(T, t)
$$


where the wave function $\psi(T, t)$ is a solution to the corresponding quantum Hamiltonian coming from the action (10), i.e. in the case where we restrict ourselves to the space-independent tachyon mode precisely (2). This should capture the most important quantum aspects of the D-brane as long as one does not consider the annihilation and creation of D-branes.

Our aim now is to determine how an open string will propagate (and e.g. eventually emit closed string radiation) in the fluctuating quantum background given by $\psi(T, t)$.

An open string moving in the classical open string background $T_{c l}(t)$ has a boundary action $^{1}$

$$
e^{i \int_{B} d \tau T_{c l}(t(\tau))}
$$

Let us recast it in the following form:

$$
e^{i \int_{t_{a}}^{t_{b}} d t T_{c l}(t)\left(\frac{1}{t_{1}}+\ldots+\frac{1}{t_{n}}\right)} \equiv e^{i \int_{t_{a}}^{t_{b}} d t T_{c l}(t) J(t)}
$$

where $t_{i}$ are the parameterizations of various sectors of the string boundary and $t_{a}$ and $t_{b}$ are the minimum and maximum time coordinates of the boundary of the string world-sheet and where we view $J(t)$ as an external driving force for $T(t)$. The natural generalization from a classical $T_{c l}(t)$ to a quantum state described by the wave function $\psi(T, t)$ is

$$
e^{i \int_{t_{a}}^{t_{b}} d t J(t) T_{c l}(t)} \rightarrow\left\langle e^{i \int_{t_{a}}^{t_{b}} d t J(t) T(t)}\right\rangle_{\psi}
$$

i.e. the expectation value of an (inverted) harmonic oscillator in the presence of an external source $J(t)$. This expectation value can (in principle) be explicitly evaluated since we know the propagator $K_{J}\left(T_{b}, t_{b} ; T_{a}, t_{a}\right)$, of the (inverted) harmonic oscillator in the presence of an external source $J(t)$. For the ordinary harmonic oscillator with cyclic frequency $\omega$ one has

$$
K_{J}\left(T_{b}, t_{b} ; T_{a}, t_{a}\right)=\left(\frac{\omega}{2 \pi i \sin \omega T}\right)^{\frac{1}{2}} e^{i\left[S_{c l}^{0}+\left(T_{a} J_{a}+T_{b} J_{b}\right)+J_{a b}\right]}
$$

where $S_{c l}^{0}\left(T_{a}, t_{a} ; T_{b}, t_{b}\right)$ is the classical action of a harmonic oscillator which at time $t_{a}$ is at position $T_{a}$ and at time $t_{b}$ at position $T_{b}$ :

$$
S_{c l}^{0}\left(T_{a}, t_{a} ; T_{b}, t_{b}\right)=\frac{\omega}{2 \sin \omega T}\left[\left(T_{a}^{2}+T_{b}^{2}\right) \cos \omega T-2 T_{a} T_{b}\right],
$$

where $T=t_{b}-t_{a}$. Finally $J_{a}, J_{b}$ and $J_{a b}$ are given by

$$
J_{a}=\int_{t_{a}}^{t_{b}} d t J(t) \frac{\sin \omega\left(t_{b}-t\right)}{\sin \omega T}, \quad J_{b}=\int_{t_{a}}^{t_{b}} d t J(t) \frac{\sin \omega\left(t-t_{a}\right)}{\sin \omega T},
$$

\footnotetext{
${ }^{1}$ Throughout the paper we are using Minkowski signature.
} 
and

$$
J_{a b}=\frac{1}{2} \int d t d t^{\prime} J(t) D_{0}\left(t, t^{\prime}\right) J\left(t^{\prime}\right)
$$

where

$$
D_{0}\left(t, t^{\prime}\right)=\theta\left(t-t^{\prime}\right) \frac{\sin \omega\left(t-t^{\prime}\right)}{\omega}-\frac{\sin \omega\left(t-t_{a}\right) \sin \omega\left(t_{b}-t^{\prime}\right)}{\omega \sin \omega T} .
$$

$D_{0}$ is the propagator corresponding to boundary conditions $T\left(t_{a}\right)=T\left(t_{b}\right)=0$. One obtains the corresponding expression for the inverted harmonic oscillator by the analytical continuation $\omega \rightarrow i \omega$.

We thus have

$$
\left\langle e^{i \int_{t_{a}}^{t_{b}} d t J(t) T(t)}\right\rangle_{\psi}=\int d T_{a} d T_{b} \psi^{*}\left(T_{b}, t_{b}\right) K_{J}\left(T_{b}, t_{b} ; T_{a}, t_{a}\right) \psi\left(T_{a}, t_{a}\right) .
$$

In the final expression explicit dependence on $t_{a}$ and $t_{b}$ should cancel and we should get a functional of $J(t)$. The expression (21) has a natural interpretation of (the exponential of) the quantum boundary action since it describes how the exponential of the classical action is changed if $T_{c l}(t) \rightarrow \psi(T, t)$.

\section{The boundary action for gaussian wave functions}

In order to exemplify the prescription let us consider a Gaussian wave packet with the initial condition

$$
\psi(T, 0)=\left(\frac{a}{\pi}\right)^{\frac{1}{4}} e^{-\frac{a}{2}\left(T-T_{0}\right)^{2}} .
$$

After a rather long calculation one obtains

$$
\left\langle e^{i \int_{t_{a}}^{t_{b}} d t J(t) T(t)}\right\rangle_{\psi}=e^{i T_{0} \int d t J(t) \cosh t+\frac{i}{2} \int d t d t^{\prime} J(t) D_{a}\left(t, t^{\prime}\right) J\left(t^{\prime}\right)}
$$

or, using the definition of $J(t)$ :

$$
\left\langle e^{i \int_{B} d \tau T(t(\tau))}\right\rangle_{\psi}=e^{i \int_{B} d \tau T_{0} \cosh t(\tau)+\frac{i}{2} \int_{B} \int_{B} d \tau d \tau^{\prime} D_{a}\left(t(\tau), t^{\prime}\left(\tau^{\prime}\right)\right)} .
$$

In (23) and (24) we used the notation

$$
D_{a}\left(t, t^{\prime}\right)=\theta\left(t-t^{\prime}\right) \frac{\sinh \omega\left(t-t^{\prime}\right)}{\omega}+i \frac{\omega^{2} \cosh \omega t \cosh \omega t^{\prime}+a^{2} \sinh \omega t \sinh \omega t^{\prime}}{2 a \omega^{2}}
$$

where we have kept $\omega$ (which should be put equal to one) in order to make clear how this is related to the corresponding expression for the ordinary harmonic oscillator by $\omega \rightarrow i \omega$. Note that the final results for the inverted and normal 
harmonic oscillators are not related by an analytical continuation. This is due to the wavefunctions appearing in (21). Eq. (25) simplifies if $a=\omega:^{2}$

$$
D_{\omega}\left(t, t^{\prime}\right)=\theta\left(t-t^{\prime}\right) \frac{\sinh \omega\left(t-t^{\prime}\right)}{\omega}+i \frac{\cosh \omega\left(t+t^{\prime}\right)}{2 \omega}
$$

One observes that indeed all explicit reference to $t_{a}$ and $t_{b}$ has dropped out and that the quantum boundary action corresponding to (23) or (24) has the structure of the classical boundary action (the term linear in $J(t)$ ) and a quantum correction (the term quadratic in $J(t)$ ). We note the explicit appearance of the classical boundary action, which is somewhat non-trivial since $T_{0}$ only appears in the position of the peak of the wave packet at $t=0$. This can be understood as follows: since the potential is quadratic we know that the expectation value $\langle T(t)\rangle$ will precisely follow a classical trajectory, and in fact it is not difficult to show that the classical trajectory associated with the wave packet $\psi(T, 0) \sim \exp (-a(T-$ $\left.T_{0}\right)^{2} / 2$ is that of a particle put at $T_{0}$ at time $t=0$ with zero momentum. Thus our choice of wave packet (22) is associated with the classical path $T_{0} \cosh \omega t$. It is also worth to note that Gaussian wave packets at a specific time $t$ remain Gaussian in $T$ at any time since the propagator is Gaussian. However, the width can change in time and the Gaussian wave packet for the inverted harmonic oscillator spreads so fast that even if the peak follows a classical trajectory, coming in from $T=\infty$ and being reflected at position $T_{0}$ at $t=0$, finally to move back to $T=\infty$, it has at any time a non-vanishing tail at the other side of the potential $V(T)=-T^{2} / 2$.

Finally note that the quantum corrections have introduced an imaginary part in the quantum boundary action. From the point of view of quantum mechanics of the (harmonic or) inverted harmonic oscillator it just reflects that the presence of a source $J(t)$ can change the wave function $\psi(T, t)$ which is a solution corresponding to the Hamiltonian (2) without the presence of $J(t)$. Eq. (23) gives the amplitude for the system to remain in state $|\psi\rangle$ after being perturbed by the external source $J$.

\section{Conformal invariance}

It is well known $[8,9,10]$ that the classical rolling tachyon boundary interaction is conformally invariant (in the sense that it defines a BCFT and provides a consistent open string background). It is therefore interesting to ask what happens when the quantum-mechanical corrections are included.

\footnotetext{
${ }^{2}$ For the ordinary harmonic oscillator it comes a function of $t-t^{\prime}$ only:

$$
D_{\omega}\left(t, t^{\prime}\right)=\theta\left(t-t^{\prime}\right) \frac{\sin \omega\left(t-t^{\prime}\right)}{\omega}+i \frac{\cos \omega\left(t-t^{\prime}\right)}{2 \omega} .
$$


We will now show that the boundary action (24) is conformally invariant at one loop. To this end we have to calculate the $\beta$ function for a boundary interaction of the form:

$$
\int d \tau T_{1}(t(\tau))+\int d \tau d \tau^{\prime} T_{2}\left(t(\tau), t\left(\tau^{\prime}\right)\right)
$$

At one loop the first term gives the standard contribution $T_{1}-d^{2} / d t^{2} T_{1}$ which obviously vanishes for the classical rolling tachyon profile. For the second term one performs an analogous calculation by decomposing $t(\tau)=t_{0}(\tau)+\xi(\tau)$, inserting it into the action (28) and expanding up to quadratic order in $\xi(\tau)$. The background field $t_{0}(\tau)$ is chosen so that the linear terms will vanish. The contractions are then made with

$$
\left\langle\xi(\tau) \xi\left(\tau^{\prime}\right)\right\rangle=2 \log \left|\tau-\tau^{\prime}\right|
$$

regularized at coinciding points as $2 \log \Lambda$. One then has to isolate terms proportional to $\log \Lambda$. One gets a straightforward generalization of the local terms:

$$
-2 T_{2}\left(t, t^{\prime}\right)+\frac{d^{2}}{d t^{2}} T_{2}\left(t, t^{\prime}\right)+\frac{d^{2}}{d t^{\prime 2}} T_{2}\left(t, t^{\prime}\right)
$$

and the $\log \Lambda$ piece of

$$
2 \frac{d}{d t} \frac{d}{d t^{\prime}} T\left(t, t^{\prime}\right)\left\langle\xi(\tau) \xi\left(\tau^{\prime}\right)\right\rangle
$$

Let us first discuss the continous part of (25). Eq. (30) then vanishes trivially, while the integral of (31) will not give a divergence as the logarithmic singularity in (29) is integrable. Hence it will not generate a contribution to the $\beta$ function. The discontinous part

$$
D_{a}^{\text {discont. }}\left(t, t^{\prime}\right)=\theta\left(t-t^{\prime}\right) \sinh \left(t-t^{\prime}\right)
$$

satisfies

$$
\left(\frac{d^{2}}{d t^{2}}-1\right) D_{a}^{\text {discont. }}\left(t, t^{\prime}\right)=\delta\left(t-t^{\prime}\right)
$$

Therefore (30) gives $2 \delta\left(t-t^{\prime}\right)$, while (31) gives

$2\left\{-2 \delta\left(t-t^{\prime}\right) \cosh \left(t-t^{\prime}\right)-\theta\left(t-t^{\prime}\right) \sinh \left(t-t^{\prime}\right)-\delta^{\prime}\left(t-t^{\prime}\right) \sinh \left(t-t^{\prime}\right)\right\}\left\langle\xi(\tau) \xi\left(\tau^{\prime}\right)\right\rangle$

Only the first and last terms will contribute to the $\beta$ function and will give $-2 \delta\left(t-t^{\prime}\right)$ which cancels the contribution of $(30)$.

From the above we see that the quantum corrections do not spoil conformal invariance at one loop. It would be very interesting to verify if the result extends to higher orders in $\alpha^{\prime}$. In fact the properties of field theories with nonlocal (bilocal) boundary interactions seems to be largely unexplored (for a first study 
see [11]), yet such theories seem to appear here quite naturally. Moreover such types of boundary interactions can have quite nontrivial properties like mimicking closed strings [12] etc. Care must also be taken, however, as there are examples of bilocal boundary interactions which are $S L(2, \mathbb{C})$ invariant and yet break full Virasoro invariance [12].

\section{The quantum decay rate}

The starting point of the calculation of the decay of the D-brane is the disk amplitude. As shown in [7] the amplitude to decay into closed strings tachyons is (in analogy with (4) and (5)) given by

$$
A(E)=\int d t e^{i E t} \rho(t)
$$

where $\rho(t)$ is obtained as ("tilde" means no zero mode)

$$
\rho(t)=\left\langle e^{i \int_{B} d \tau T_{0} \cosh \left(t+\tilde{X}_{0}(\tau)\right)}\right\rangle_{d i s k^{\prime}}
$$

The corresponding expression for a quantum D-brane is now obtained from (24) by the replacement

$$
e^{i T_{0} \int_{B} d \tau \cosh \left(t+\tilde{X}_{0}(\tau)\right)} \rightarrow e^{i S^{(q b a)}\left(t+\tilde{X}_{0}\right)}
$$

where the quantum boundary action $S^{(q b a)}\left(t+\tilde{X}_{0}\right)$ is

$$
T_{0} \int_{B} d \tau \cosh \left(t+\tilde{X}_{0}(\tau)\right)+\frac{1}{2} \int_{B} \int_{B} d \tau_{1} d \tau_{2} D_{a}\left(t+\tilde{X}_{0}\left(\tau_{1}\right), t+\tilde{X}_{0}\left(\tau_{2}\right)\right)
$$

We thus have a modified $\rho(t)$, much along the lines of the $2 \mathrm{~d}$ case:

$$
\rho_{\text {quan }}(t)=\left\langle e^{i S^{q b a}\left(t+\tilde{X}_{0}\right)}\right\rangle_{d i s k^{\prime}}=\int \mathcal{D} \tilde{X}_{\mu} e^{i S^{q b a}\left(t+\tilde{X}_{0}\right)} e^{i \int_{d i s k} d^{2} z \partial \tilde{X}_{\mu} \partial \tilde{X}^{\mu}},
$$

and a modified emission amplitude

$$
A_{\text {quan }}(E)=\int d t e^{i E t} \rho_{\text {quan }}(t)
$$

In principle one can just apply the substitution (37) and perform the functional average in the calculation of $\rho_{\text {quan }}(t)$. The simplest way to do this was outlined in [6] for the classical solution $T_{c l}(t)=T_{0} e^{t}$ (the half-brane). However, the method can also be used for $T_{c l}(t)=T_{0} \cosh t$. Technically it is somewhat more complicated since one will encounter contraction of exponentials $e^{\tilde{X}_{0}\left(\tau_{1}\right)}$ and $e^{-\tilde{X}_{0}\left(\tau_{2}\right)}$, and these result in singularities when points coincide on the boundary. However, 
the regularization proposed in [9] allows one to reproduce the result first derived by Sen [4]

$$
\rho(t)=\frac{1}{1+\sin \left(\pi T_{0}\right) e^{t}}+\frac{1}{1+\sin \left(\pi T_{0}\right) e^{-t}}-1 .
$$

We mention this because one encounters no further technical obstacles when using the substitution (37) in the calculation (39).

Unfortunately we have been unable to perform the calculation explicitly to a level that a formula like (41) appears, i.e. expand the exponential $e^{i S^{(q b a)}\left(t+\tilde{X}_{0}\right)}$, do all the Wick contractions of the exponentials and sum the corresponding series. But we note the following: the qualitative form of $\rho(t)$ can be understood without calculations: the fast oscillations of $e^{i \int_{B} d \tau \cosh (t+\tilde{X}(\tau))}$ caused $\rho(t)$ to vanish for $t \rightarrow \pm \infty$. The same is likely to be true for $\rho_{\text {quan }}(t)$ since in addition we have an exponentially decaying part in (37). However, the implications for the decay amplitude $A_{\text {quan }}(E)$ given by (40) is less clear since the integral for large $E$ will be dominated by the pole closest to the real axis. This is the reason it is not enough to perform an expansion of $\rho_{\text {quan }}(t)$ in powers of $e^{t}$ to finite order (which can indeed be done, as mentioned). One needs more precise information in order to determine the large $E$ behavior of the Fourier transform $A_{\text {quan }}(E)$.

Thus so far we cannot state that emission of large energy closed string states is damped by the quantum nature of the tachyon as was the case in $2 \mathrm{~d}$ string theory, although it is likely to be true. In any case it would be very interesting to perform such a calculation.

\section{Inclusion of momentum modes}

So far we have restricted ourselves to tachyons with no momentum dependence (the tachyon zero-mode). Let us now examine how the quantum treatment of the momentum modes influences the picture.

As we have already seen in formula (10), we obtain a set of inverted harmonic oscillators (with $k^{2}<1$ ) and a set of normal ones (modes with $k^{2}>1$ ). A natural wave function to consider is a Gaussian wave packet (with $a=|\omega|$ ) for each of the modes. For simplicity we set the initial 'position' $T_{0}$ to zero. This kind of wave function illustrates the weirdness of dealing with tachyons: the higher and lower modes behave completely differently - the higher modes are just in their stationary vacuum state while the lower ones spread out due to the inverted harmonic potential.

The boundary action for an open string is given by (8) so we have to calculate

$$
S_{b}^{\text {quantum }}=\prod_{\vec{n}}\left\langle e^{\int d \tau \frac{1}{(2 \pi L)^{\frac{p}{2}}} \phi_{\bar{n}}^{c}(t) \cdot \cos k x}\right\rangle_{\psi} \cdot\left\langle e^{\int d \tau \frac{1}{(2 \pi L)^{\frac{p}{2}}} \phi_{\bar{n}}^{s}(t) \cdot \sin k x}\right\rangle_{\psi}
$$


The result follows from (26) and (27). For the higher, normal (cosine) modes one obtains

$$
e^{\frac{i}{2} \int d \tau d \tau^{\prime} \frac{1}{(2 \pi L)^{p}} \cos k x \cos k x^{\prime}\left[\theta\left(t-t^{\prime}\right) \frac{\sin \omega\left(t-t^{\prime}\right)}{\omega}+\frac{i}{2} \frac{\cos \omega\left(t-t^{\prime}\right)}{\omega}\right]}
$$

where $\omega=\sqrt{k^{2}-1}$. The result for the sine modes is similar. Note that (43) contains no dependence on the time $t$ which enters into the calculation of $\rho_{\text {quan }}(t)$ in accordance with the fact that we are just using the standard vacuum wave functions for the higher modes.

For the lower, tachyonic modes we obtain from (27):

$$
e^{\frac{i}{2} \int d \tau d \tau^{\prime} \frac{1}{(2 \pi L)^{p}} \cos k x \cos k x^{\prime}\left[\theta\left(t-t^{\prime}\right) \frac{\sinh \kappa\left(t-t^{\prime}\right)}{\kappa}+\frac{i}{2} \frac{\cosh \kappa\left(t+t^{\prime}\right)}{\kappa}\right]}
$$

with $\kappa=\sqrt{1-k^{2}}$.

Now we have to perform a summation over $k$ in the exponential. The volume factor and summation transforms into an integral, while the $\omega$ in the denominator gives the standard Lorentz-invariant phase space measure. The dominant behavior for large $t, t^{\prime}$ comes from second piece in the formula for the lower, tachyonic modes. We thus have to evaluate:

$$
-\frac{1}{4} \int_{k^{2}<1} \frac{d^{p} k}{\sqrt{1-k^{2}}} \cos \left(\vec{k} \cdot\left(\vec{x}-\overrightarrow{x^{\prime}}\right)\right) \cosh \left(\sqrt{1-k^{2}}\left(t+t^{\prime}\right)\right)
$$

This integral can be performed analytically in the small $\left|x-x^{\prime}\right|$ region and we obtain

$$
\text { const } \cdot \frac{I_{\frac{p-1}{2}}\left(t+t^{\prime}\right)}{\left(t+t^{\prime}\right)^{\frac{D-2}{2}}}
$$

For large $t, t^{\prime}$ this behaves exponentially like the zero mode (in fact it is the dominant zero mode which gives this contribution) - so the qualitative features of the result, like the exponential suppression of the partition function/amplitudes at large times, is similar as in the zero-mode case and we do not expect any additional surprises coming from the space-dependent tachyonic modes.

\section{Discussion}

As mentioned in the introduction the considerations in this paper were motivated on the one hand by the desire to understand more about the nature of quantum D-branes, and on the other hand by the very explicit quantum effects which could be analyzed quantitatively for D0-branes in $2 \mathrm{~d}$ string theory [5]. Some generic aspects have emerged from this investigation.

The first is that the quantum nature $T_{c l}(t) \rightarrow \psi(T, t)$ makes it impossible to ignore the "other side" of the potential $V(T)=-T^{2} / 2$. As discussed above the wave packets always have a tail on the other side of the potential. This is bad 
since the "other side" of the full tachyonic potential in bosonic string theory is unbounded from below like $T^{3}$. This is much worse than $-T^{2}$. There is no natural self-adjoint Hamiltonian in such a potential and most likely there is no cure for such a theory. It makes the role of the conjectured minimum on the "right side" of the potential somewhat dubious. Of course one can alway confine oneself to the study of superstrings where the potential is bounded from below.

Secondly, the quantum correction to the boundary action seems to preserve conformal invariance at least to one loop order. It would be very interesting to study these issues in more detail.

Thirdly, there will generically be an imaginary part in the quantum boundary action. For the simplest constant mode wave function (22) with $a=1$ in Minkowski space one has a term

$$
e^{-\frac{1}{4} \int d \tau \int d \tau^{\prime} \cosh \left(2 t+X_{0}^{\prime}(\tau)+X_{0}^{\prime}\left(\tau^{\prime}\right)\right)}
$$

This very strong suppression of "propagation" for large times seems to be a genuine quantum effect related to the state $\psi(T, t)$ and such behavior is characteristic for potentials unbounded from below like the inverse harmonic potential.

The appearance of this term has an interesting consequence with regards to the conjecture that in the tachyon vacuum open strings disappear and only closed strings are left. The amplitude for an open string propagating to very large times will always be exponentially supressed with respect to the amplitude of an open string transforming into a closed string at some finite time, even though the latter amplitude would be penalized by a factor of $g_{s}$.

Finally let us note that it would also be very interesting to explore the role of quantum backgrounds for closed strings, not necessarily just in the context of tachyonic physics.

\section{Acknowledgment}

J.A. and R.J. acknowledge support by the EU network on "Discrete Random Geometry", grant HPRN-CT-1999-00161. and by "MaPhySto", the Center of Mathematical Physics and Stochastics, financed by the National Danish Research Foundation. RJ was supported in part by KBN grants 2P03B09622 (2002-2004), 2P03B08225 (2003-2006) and 1P03B02427 (2004-2007).

\section{References}

[1] W. Taylor and B. Zwiebach, D-branes, tachyons, and string field theory, arXiv:hep-th/0311017. 
[2] I. R. Klebanov, J. Maldacena and N. Seiberg, D-brane decay in twodimensional string theory, JHEP 0307 (2003) 045, [arXiv:hep-th/0305159].

[3] J. McGreevy, J. Teschner and H. Verlinde, Classical and quantum D-branes in 2D string theory, JHEP 0401 (2004) 039, [arXiv:hep-th/0305194].

[4] A. Sen, Rolling tachyon, JHEP 0204, 048 (2002), [arXiv:hep-th/0203211].

[5] J. Ambjorn and R. A. Janik, The decay of quantum D-branes, Phys. Lett. B 584, 155 (2004), [arXiv:hep-th/0312163].

[6] F. Larsen, A. Naqvi and S. Terashima, Rolling tachyons and decaying branes, JHEP 0302, 039 (2003), [arXiv:hep-th/0212248].

[7] N. Lambert, H. Liu and J. Maldacena, Closed strings from decaying D-branes, arXiv:hep-th/0303139.

[8] J. Polchinski and L. Thorlacius, Free fermion representation of a boundary conformal field theory, Phys. Rev. D 50 (1994) 622 [arXiv:hep-th/9404008].

[9] C. G. . Callan, I. R. Klebanov, A. W. W. Ludwig and J. M. Maldacena, Exact solution of a boundary conformal field theory, Nucl. Phys. B 422, 417 (1994), [arXiv:hep-th/9402113].

[10] A. Recknagel and V. Schomerus, Boundary deformation theory and moduli spaces of D-branes, Nucl. Phys. B 545, 233 (1999) [arXiv:hep-th/9811237].

[11] K. Li and E. Witten, Role of short distance behavior in off-shell open string field theory, Phys. Rev. D 48, 853 (1993) [arXiv:hep-th/9303067].

[12] J. Ambjorn and R. A. Janik, Interpolating between open and closed strings - a BSFT approach, Phys. Lett. B 538, 189 (2002) [arXiv:hep-th/0203185]. 